\title{
LOCK-IN THERMOGRAPHY INVESTIGATION OF SHUNTS IN SCREEN-PRINTED AND PERL SOLAR CELLS
}

\author{
Otwin Breitenstein, Jean P. Rakotoniaina, Sven Neve, Martin A. Green*, Jianhua Zhao*, Aihua Wang*, and Giso Hahn** \\ Max Planck Institute of Microstructure Physics, Weinberg 2, D-06120 Halle, Germany \\ ${ }^{*}$ Centre for Photovoltaic Engineering, University of New South Wales, Sydney 2052, Australia \\ ** University of Konstanz, PF X916, D-78457 Konstanz, Germany
}

\begin{abstract}
We compare the application of highly sensitive infrared (IR) lock-in thermography for the investigation of local shunts in multicrystalline screen-printed solar cells and in high-efficiency PERL cells made from CZ-Si. In both cell types local shunts are found, showing a nonlinear (diode-like) I-V characteristic. If a reverse bias as large as $13 \mathrm{~V}$ is applied, a number of additional hot spots appear in all cells. In the multicrystalline cells, some of these hot spots may be connected with crystal defects, but most of the shunts dominating under forward bias are due to technological imperfections. One of the PERL cells was completely free of shunts acting under forward bias. Only this cell showed an I-V characteristic without any "second diode" contribution. This is a proof that the "second diode" current in these and probably also in most other solar Si cells is essentially due to local shunts.
\end{abstract}

\section{INTRODUCTION}

Lock-in thermography has been proven to be a valuable tool to investigate shunts in screen-printed CZ solar cells [1], in floating pn-junctions [2], and in multicrystalline cells [3]. In this contribution this technique is also applied to highly efficient "passivated emitter, rear bcally-diffused" (PERL) solar cells. Lock-in thermography is a highly sensitive technique to image local heat sources in electronic devices. A lock-in thermography system, which is specialized to the investigation of shunts and other local heat sources in electronic devices is commercially available [4]. In this technique a pulsed bias is applied to a device in the dark, and the temperature modulation at the surface is imaged with a highly sensitive infrared thermocamera. The data are evaluated by numerical lock-in processing and averaged over a certain acquisition time. The advantage of lock-in thermography over stationary one is a dramatically improved detection sensitivity (from $20 \mathrm{mK}$ to $<100 \mu \mathrm{K}$ ) and a considerably improved spatial resolution, since the lateral heat spreading is largely suppressed in this a.c. technique.

\section{EXPERIMENTAL}

The $8 \times 10 \mathrm{~cm}^{2}$ sized screen-printed cells have been manufactured at University of Konstanz on BAYSIX material. They were manufactured using a standard cell process implying $\mathrm{NaOH}$ etching, $\mathrm{POCl}_{3}$ diffusion, $\mathrm{SiN} \mathrm{AR}$ coating, screen printed front contact, screen printing back contact, cofiring, and wafer saw edge isolation. The $2 \times 2 \mathrm{~cm}^{2}$ sized PERL cells have been manufactured at UNSW Sydney on Czochralski (CZ) material using the cell process described elsewhere [5]. The lock-in thermography investigations have been performed using the TDL 384 M 'Lock-in' system made by Thermosensorik $\mathrm{GmbH}$ Erlangen [4]. All thermograms shown are amplitude images taken at $3 \mathrm{~Hz}$ lock-in frequency. For every cell three measurements were performed: One in forward direction near the bias of the maximum power point of the cells $(0.5 \ldots 0.53 \mathrm{~V})$, revealing the shunts which degrade $V_{o c}$ and the fill factor, one in reverse direction at the same bias in order to check the symmetry of the I-V characteristics of the shunts, and one at $-13 \mathrm{~V}$ reverse bias in order to detect hot spots, which may become dangerous for the module if a cell is shaded [6].

\section{RESULTS}

\section{Screen printed cells}

In Fig. 1 thermography results of two screen-printed cells SP1 and SP2 are shown for the three measurement conditions with the corresponding scaling ranges indicated in the caption. These cells are fabricated from neighbouring wafers in the block, hence their crystal structure is nearly the same in both cells. Any shunting caused by extended grown-in crystal defects in the material should be visible in both cells at the same position. The measurements at $0.5 \mathrm{~V}$ bias took $1 / 2 \mathrm{hr}$ each, whereas the $-13 \mathrm{~V}$ reverse bias measurements took only $1 \mathrm{~s}$, since the signal was considerably stronger here. In Fig. 1 (a) we see a strong local shunt with an extended bow-shaped appearance, which does not appear under reverse bias. This shunt appears artificially 

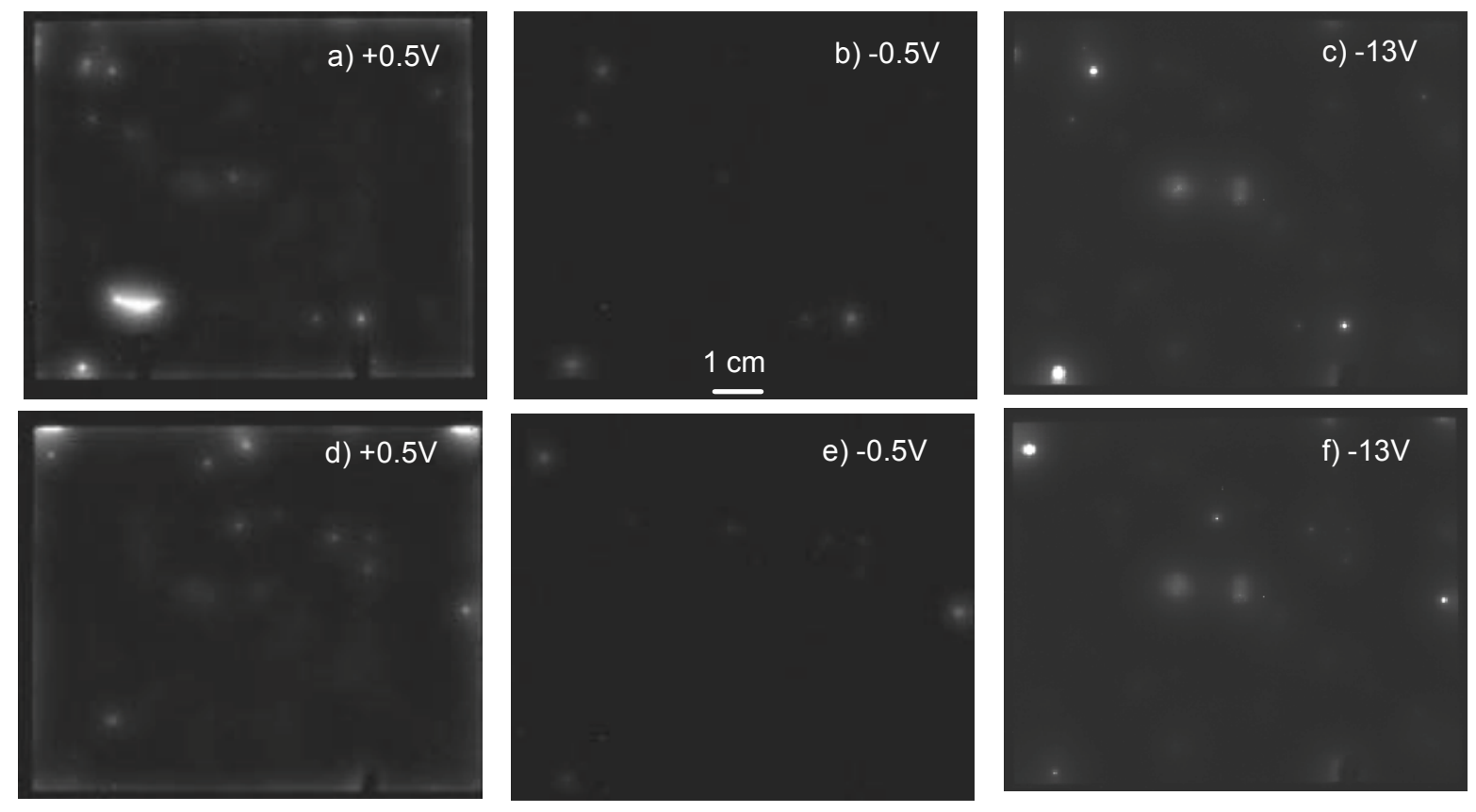

Fig. 1: Lock-in thermograms of two screen-printed solar cells. (a) SP1, +0.5 V forward, $3 \mathrm{mK}$; (b) SP1, $-0.5 \mathrm{~V}$ reverse, 3 $\mathrm{mK}$; (c) SP1, -13 V reverse, $500 \mathrm{mK}$, (d) SP2, +0.5 V forward, $3 \mathrm{mK}$; (e) SP2, -0.5 V reverse, $3 \mathrm{mK}$, (f) SP2, -13 V reverse, $500 \mathrm{mK}$

broadened in Fig. 1 (a), since its signal height was beyond the scaling range. At this position there is an indication of a weak scratch at the surface. Moreover, there are some more shunts in the area of both cells and in their edge region. All shunts in Fig. 1 appear more or less weaker at $-0.5 \mathrm{~V}(\mathrm{~b}, \mathrm{e})$ than at $+0.5 \mathrm{~V}(\mathrm{a}, \mathrm{d})$, hence they have a more or less diode-like characteristic with the larger current flowing under forward bias of the cell. Especially, the edge shunts generally do not appear under reverse bias. Only some of the shunts visible in forward direction are leading to hot spots under $-13 \mathrm{~V}$. On the other hand, most but not all of the hot spots visible under $-13 \mathrm{~V}$ also appear at $+0.5 \mathrm{~V}$. The two extended ones in the middle and some other shunts areappearing under $-13 \mathrm{~V}$ in both cells in the same position. Hence, they are probably connected with extended crystal defects in the material. The dominant shunts in forward direction, however, are not correlated in both cells, hence they are probably not induced by the crystal structure but rather by technological imperfections. These findings generally agree with other investigations performed on similar cells [3]. Especially, it turns out again that shunts observed under large reverse bias are usually not the dominant shunts at the working point in forward bias. This statement, however, holds only in the absence of ohmic shunts, which dominate both in forward and in reverse direction.

\section{PERL cells}

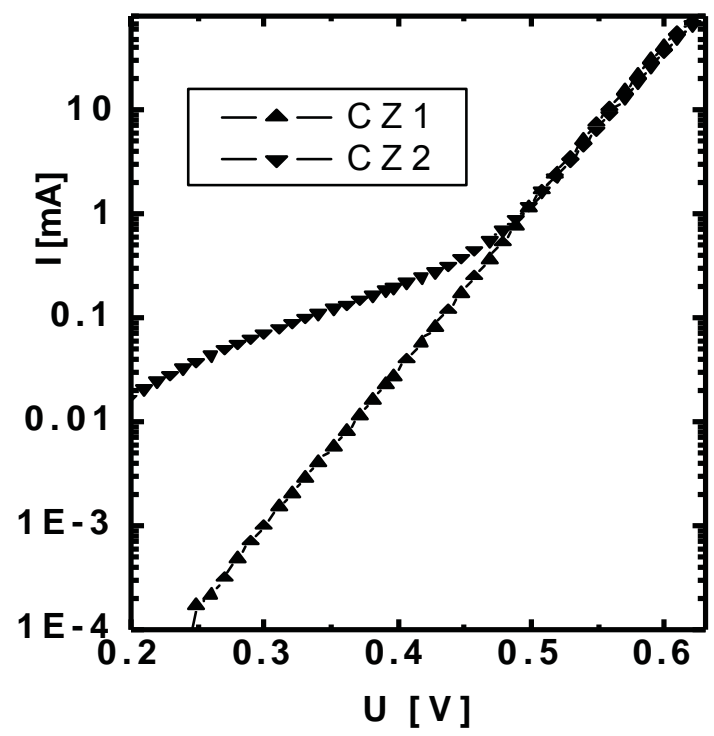

Fig. 2: Dark I-V characteristics of the two PERL cells

Fig. 2 shows the I-V characteristics of the two PERL cells CZ1 and CZ2, which clearly differ from each other. Only CZ1 shows an exponential characteristic over a large bias range with an $n$-factor of about 1.1 , but the low voltage part of CZ2 contains a pronounced "second diode" current contribution with an n-factor above 3 . In 

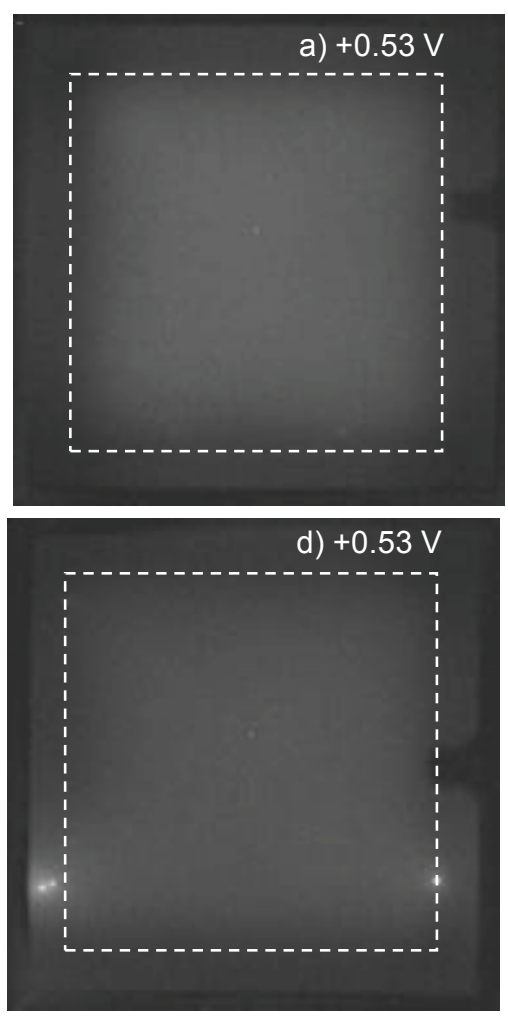
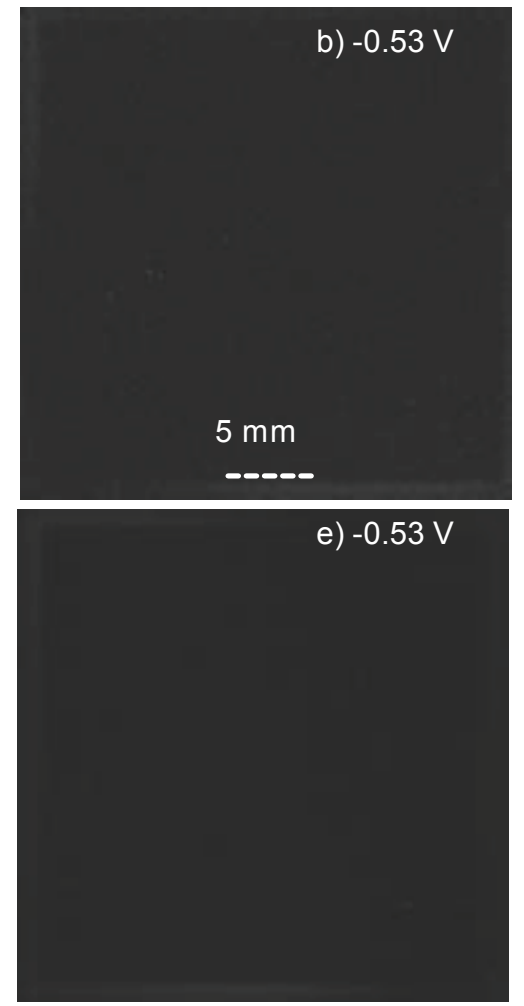
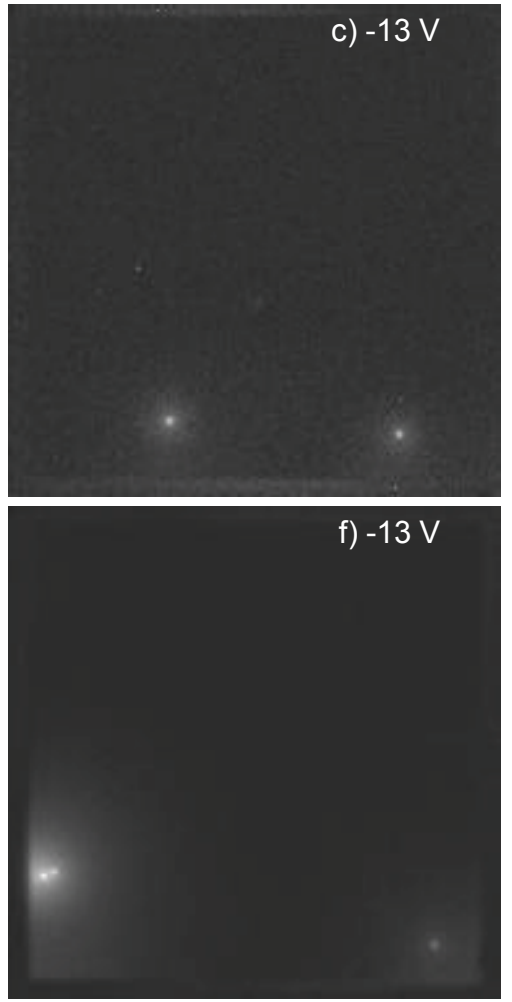

Fig. 3: Lock-in thermograms of two PERL solar cells. (a) CZ1, +0.53 V forward, $1 \mathrm{mK}$; (b) CZ1, $-0.53 \mathrm{~V}$ reverse, $1 \mathrm{mK}$; (c) CZ1, $-13 \vee$ reverse, $0.5 \mathrm{mK}$ (!), (d) CZ2, +0.53 V forward, $1 \mathrm{mK},(\mathrm{e}) \mathrm{CT} 2,-0.53 \mathrm{~V}$ reverse, $1 \mathrm{mK}$, (f) CZ2, $-13 \mathrm{~V}$ reverse, $130 \mathrm{mK}$

fact, the latter type of I-V characteristic is typical for most solar cells. One question was: Can lock-in thermography help to find the origin of this difference?

The corresponding lock-in thermography results of the two PERL cells CZ1 and CZ2 are shown in Fig. 3. The forward bias has been chosen at $0.53 \mathrm{~V}$ here, since the open circuit voltage of these cells was higher than that of the screen-printed cells. It should be mentioned that the active area of these cells (marked dashed in (a) and (d)) is smaller than the wafer size. Their active area is surrounded by an oxidized region, which carries a ring- shaped metal contact. In Fig. 3 there is no indication of an extra leakage current in the edge region, which was clearly observed under forward bias in Figs. 1 (a) and (d). This is a direct consequence of the fact that in the PERL cells the edge of the pn-junction is lying wellpassivated below the oxide, whereas in the screenprinted cells it crosses the sawed wafer edge. Cell CZ1 does not show any local shunts in forward direction and only very weak ones in reverse direction (note the different scaling ranges!). In cell CZ2, however, some local shunts are clearly visible in forward direction in the lower region of (d). These shunts are not visible at -0.53 $\checkmark$ reverse bias (e), hence they show a highly non-linear I-V characteristic. Only the left ones become hot spots under $-13 \vee$ reverse bias (f). Moreover, under $-13 \vee$ an- other weak hot spot appears, which was not visible under forward bias. All these shunts are lying below or near to the ring-shaped metal contact. Those below this contact probably can be identified as holes in the oxide, leading to a Schottky-type shunt below the metal ring contact. The shunts in the area are most probably sites of a locally strong recombination action in the space charge layer, which also would be active preferably under forward bias and also could lead to pre-breakdown sites. These defects could originate e.g. from scratches made by attempts to put on a contact needle.

The shunts visible in Fig. 3 (d) are obviously responsible for the degraded I-V characteristic of cell CZ2, which was shown in Fig. 2. Also this behaviour corresponds to our earlier observation that the whole lowbias part of most silicon solar cells is completely due to local shunts, whereas the shunt-free regions of the cells behave as a very ideal pn-junction [3]. Obviously cell CZ1 is one of the rare examples of a completely shuntfree cell. Compared to usual cells this cell should show an extraordinary efficiency under low-illumination conditions. Indeed, under weak room light conditions cell CZ2 showed an open circuit voltage of only $0.277 \mathrm{~V}$, whereas $\mathrm{V}_{\mathrm{oc}}$ of cell CZ1 was about $0.416 \mathrm{~V}$ under the same conditions. 


\section{CONCLUSIONS}

It has been shown that lock-in thermography may give valuable results not only for screen printed multicrystalline solar cells but also for high-efficiency PERL cells. Especially, local shunting sites, which degrade the low illumination intensity performance, can also be identified. The only cell, which was totally free of local shunts visible under forward bias, also showed an I-V characteristic being totally free of the "second diode" current contribution, which is usually present in all solar cells. This cell also shows an excellent low light level performance. This result proves that the "second diode" current of the other cells is essentially due to the presence of local shunts with a nonlinear (diode-like) I-V characteristic. It should be reminded that not all solar cells are showing solely non-linear shunts as the cells of this investigation did. Whenever shunts with a linear (ohmic) characteristic are present, they usually dominate both the forward and reverse characteristic and are even more dangerous to the low illumination performance than non-linear shunts.

\section{Acknowledgement}

This work has been supported by the German federal ministry of education and research under contract No. 0329743 B and by the Alexander von Humboldt Stiftung, Bonn.

\section{References}

[1] O. Breitenstein, M. Langenkamp, O. Lang, and A. Schirrmacher, "Shunts due to Laser Scribing of Solar Cells Evaluated by Highly Sensitive Lock-in Thermography", Solar Energy Materials \& Solar Cells 65 (2001) pp. 55-62.

[2] O. Breitenstein, M. Langenkamp, K.R. Mclntosh, C.B. Honsberg, and M. Rinio, "Localization of Shunts Across the Floating Junction of DSBC Solar Cells by Lock-in Thermography", 28th IEEE PVSC, 2000, pp. 124-127.

[3] O. Breitenstein, M. Langenkamp, J.P. Rakotoniaina, J. Zettner, "The Imaging of Shunts in Solar Cells by Infrared Lock-in Thermography", 17th Eur. PVSEC, Munich, 2001, paper VC1/26, proceedings in print.

[4] www.thermosensorik.com

[5] J. Zhao, A. Wang and M.A. Green, "24.5\% Efficiency PERT Silicon Solar Cells on SEH MCZ Substrates and Cell, Performance on other SEH CZ and FZ Substrates", Solar Energy Materials \& Solar Cells, 66, 2001, pp. 2736.

[6] W. Herrmann, M.C. Alonso, W. Boehmer, and K. Wambach, "Effective Hot-Spot Protection of PV ModulesCharacteristics of Crystalline Silicon Cells and Conse- quences for Cell Production", 17th Eur. PVSEC, Munich, 2001, paper VC1/64, proceedings in print. 